\title{
El apoyo continuo durante el parto mejora los resultados perinatales
}

\section{Objetivo}

Evaluar los efectos del apoyo o soporte continuo durante el parto en madres y bebés.

\section{Fuente de datos \\ Registro de Ensayos Clínicos Cochrane hasta Octubre del 2001.}

\section{Criterios de Selección}

Ensayos Clínicos aleatorizados, que comparan el apoyo continuo durante el trabajo de parto y el parto con el cuidado habitual.

\section{Resultados Principales}

Se incluyeron 14 ensayos clínicos, que incluyeron más de 5000 mujeres.

El soporte continuo, redujo la necesidad de analgesia farmacológi- ca en un $29 \%$ (OR 0,71 IC 95\% 0,2-0,81), el parto vaginal operativo (fórceps o extracción por vacío) en un $23 \%$ (OR 0,77 IC 95\% 0,65-0,90), la cesárea en un $23 \%$ (OR 0,77 IC 95\% 0,64-0,91), y el puntaje de Apgar@ menor a 7 a los cinco minutos, en un $50 \%$ (OR 0,50 IC 95\% 0,28-0,87).

El soporte continuo también se relacionó con una leve reducción en la duración del trabajo de parto.

Seis ensayos clínicos evaluaron la percepción de las madres con respecto a sus experiencias durante el parto, y los resultados favorecieron la estrategia comentada.

\section{Conclusiones}

El apoyo continuo durante el trabajo de parto, realizado por enfermeras, parteras o personas legas, tiene beneficios para las madres y sus bebés, y no parece tener ningún efecto dañino.

\section{Comentario}

El apoyo, soporte o asistencia continua durante el trabajo de parto incluye el consejo e información a la mujer, la asistencia tangible, es decir que ella sienta que es atendida cuando lo necesita; y el apoyo emocional, lo que significa que los asistentes puedan escucharla, tranquilizarla, consolarla y contenerla. Continuo significa que está presente durante todo el trabajo de parto y el parto. Este tipo de soporte es provisto por enfermeras, parteras o personas legas, comúnmente mujeres especialmente entrenadas o "doulas". En Sudamérica actualmente hay centros que organizan cursos de formación más sistemática sobre estos aspectos.

El Dr. José Belizán, Director del Centro Latinoamericano de Perinatología (CLAP), en su conferencia dictada en el Congreso de Medicina Perinatal realizado en Barcelona en septiembre del 2001, citó una encuesta realizada en Colombia, Argentina y Uruguay, donde se evidenciaba que el $94 \%$ de las mujeres estaban solas durante el trabajo de parto y el parto.

Ese mismo año el Parlamento uruguayo aprobó una ley que establece el derecho de toda mujer a recibir compañía durante su trabajo de parto y parto.

La disminución del $23 \%$ en las cesáreas, no es un dato menor para nuestra región. Según un estudio publicado 19991, entre Argentina, Brasil, Chile y Uruguay, se realizan anualmente más de 535.000 cesáreas innecesarias. Se consideraron como innecesarias, a la cantidad de cesáreas que supera el punto de corte aceptable para la OMS del $15 \%$ para el total de los nacimientos. En relación a este punto, se ha encontrado cierta asociación entre el parto por cesárea y un mayor riesgo de mortalidad perinatal ${ }^{2}$.

En el 2001 se corroboró en forma precisa ${ }^{3}$ que el puntaje de Apgar a los cinco minutos es un claro predictor de mortalidad neonatal. Concretamente los niños de término con un puntaje de Apgar entre 4 y 6 a los cinco minutos tienen 53 veces más riesgo de muerte durante los primeros 28 días que los niños que nacen con un Apgar de 7 o más (RR 53 [IC95\% $20-140]$ ); y los recién nacidos con Apgar de 3 o menos, tienen 1460 veces más riesgo de muerte (RR 1460 [IC95\% 835 - 2555]).

También sabemos que en algunas zonas de nuestro país y en países vecinos, no es sencillo conseguir que el trabajo de parto y parto se desarrollen asistidos por una institución o personal habilitado a tal efecto. Por ejemplo, en Bolivia en $1996^{1}$ la tasa de partos institucionales era menor al $30 \%$, asociándose a una muy alta tasa de mortalidad materna y perinatal. Esta falta de institucionalización en la atención del parto se debe a múltiples factores. Uno de ellos es que las mujeres encuentran grandes diferencias entre la atención hospitalaria y sus costumbres tradicionales en relación al momento del nacimiento, sobre todo en zonas donde imperan bagajes culturales fuertes. Una actitud más respetuosa, atenta e individualizada, como el soporte continuo, podría disminuir esta resistencia a solicitar asistencia para el nacimiento. Comúnmente las zonas donde predomina este problema son rurales, y están cubiertas por hospitales pequeños, con una baja frecuencia de partos, en donde sólo haría falta formar al personal para este tipo de apoyo; distinto es lo que ocurre en los hospitales de mayor complejidad, en donde generalmente no se cuenta con la suficiente cantidad de personal para ofrecer soporte continuo en forma individual a la totalidad de las parturientas y sus familias.

\section{Conclusiones del comentador}

El apoyo o soporte continuo es una herramienta útil en la atención del trabajo de parto y el parto, y podría mejorar sustancialmente la calidad y los resultados de la atención materno infantil.

@ El puntaje de Apgar surge de la suma de cinco características que presentan los bebés en el momento del nacimiento: frecuencia cardíaca, esfuerzo respiratorio, tono muscular, reflejos y color. Cada una de ellas puede valer 0 a 2 , según una calificación que las evalúa independientemente; finalmente se suman y se establece un puntaje total. Un Apgar de 7 o más a los 5 minutos del nacimiento indica que el recién nacido está en muy buenas condiciones.

\section{Dr. Fernando R. Vázquez Peña [ Unidad de Medicina Familiar y Preventiva. Hospital Italiano de Buenos Aires. ]}

Vázquez Peña F. El apoyo continuo durante el parto mejora los resultados perinatales. Evid. actual. práct. ambul 2004;7:135. Comentado de Hodnet ED y cols. Caregiver support for women during childbirth. Cochrane Database Syst Rev 2002; (1):CD000199.

\section{Referencias}

1.Rates and implications of caesarean sections in Latin America: ecological study. J. Belizan, F. Althabe, F. Barros, S. Alexander.BMJ 27 November $1999 ;$ Volume $319: 1397$ - 1400 2.Early neonatal mortality and cesarean delivery in México City. J. Bobadilla, G. Walker.Am J Obstet Gynecol 1991; 164:22-83

3.The Continuing Value of the Apgar Score for the assessment of newborn infants. Casey B, Mclntire D, Leveno K.N Eng J Med 2001 Feb 7; 344 : 467 - 471 . Ver el artículo comentado en castellano, en la Revista Evidencia en Atención Primaria, Volumen 4 № 5 Sept-Octubre 2001 - Pag. 139. 